\title{
Towards High Resolution in Cryo-Electron Tomography Subtomogram Analysis
}

\author{
Stefan Pfeffer ${ }^{1}$, Maryam Khoshouei ${ }^{1}$, Radostin Danev ${ }^{1}$ and Friedrich Förster ${ }^{1,2}$ \\ 1. Max-Planck Institute of Biochemistry, Department of Molecular Structural Biology, Martinsried, \\ Germany \\ 2. Cryo-Electron Microscopy, Bijvoet Center for Biomolecular Research, Utrecht University, Utrecht, \\ The Netherlands
}

Cryo-electron tomography allows three-dimensional imaging of macromolecular complexes in a closeto-native state at molecular resolution [1,2]. Due to specimen damage imposed by the imaging electrons, the signal-to-noise-ratio in cryo-electron tomography data is typically very low. This low signal-to-noise-ratio restricts interpretability of raw cryo-electron tomograms, but can ultimately be overcome by averaging many subtomograms depicting the same macromolecular complex [2-4]. Here, we introduce several approaches that have been useful to deal with the low signal-to-noise ratio and to overcome other resolution limiting factors in subtomogram analysis that mostly originate from tilting of the specimen during data acquisition.

For reconstruction of three-dimensional densities in cryo-electron tomography, individual projection images are required that depict the same specimen area from different viewing angles. In practice, this is achieved by sequential tilting of the specimen in the transmission electron microscope from approximately $-60^{\circ}$ to $60^{\circ}$ in $2^{\circ}$ increments and image acquisition at each tilt. These tilt images are then aligned with respect to a common coordinate system using interactively located gold fiducial markers evenly spread in the vitreous ice. Lastly, the aligned tilt images have to be frequency-weighted before they can be backprojected into a volume. Electron micrographs acquired at high specimen tilts suffer from several resolution-limiting factors. With increasing specimen tilt, sample thickness increases in a cosine-like manner and, as a consequence, the signal-to-noise ratio of tilt images decreases significantly. Furthermore, specimen tilt introduces a defocus gradient perpendicular to the tilt axis, which is increasingly difficult to correct accurately for at high tilt images. During tilt series acquisition, the accumulating dose, usually in the range of $60-100 \mathrm{e}^{-} / \mathrm{A}^{2}$, causes significant beam damage to the specimen. Since all tilt schemes intended for high-resolution subtomogram analysis start at low tilt, where the signal-to-noise ratio is best, the high tilt images usually have suffered most structural damage. Beam damage often also results in local deformations of the sample during tilt series acquisition. As a consequence, high-tilt images often cannot be aligned accurately with respect to the common coordinate system of the tomogram anymore.

While the effect of accumulated sample damage due to the imaging electrons can be compensated for using frequency-dependent B-factor weighting for the individual tilt images [5], the local deformation of sample geometry is difficult to correct for. A practical way to circumvent all these resolution-limiting factors inherent to high tilt images is to exclude them from final subtomogram reconstruction altogether. We demonstrate the feasibility of this approach by obtaining several subtomogram averages at subnanometer resolution and show that exclusion of high-tilt images is crucial for reaching this resolution regime. By optimizing the tilt image alignment locally for each individual gold fiducial marker, resolution of the subtomogram averages can be improved even further. In particular, we analyze mammalian ribosomal complexes either in isolation [6] or bound to the protein transport machinery of the endoplasmic reticulum membrane [7], resolving transmembrane helices of the involved components. 
Analyzing the protein transport machinery in a native membrane environment and in complex with its native interaction partners, which is only possible using cryo-electron tomography and subtomogram analysis, proves essential for capturing the 'correct' conformational state for one of the membrane embedded constituents of the protein transport machinery.

Increased signal-to-noise ratio for low spatial frequencies in the raw data promises to be beneficial for subtomogram analysis in many respects. Better low-frequency contrast increases fidelity of automatic particle localization, e.g. by template matching [8], improves particle sorting via subtomogram classification approaches and increases the accuracy of subtomogram alignment. The Volta phase plate has emerged as an effective means to improve low-frequency contrast in cryo-electron microscopy imaging modalities [9] by introducing a relative phase shift between the scattered and unscattered electrons. This allows imaging of biological specimens in or close-to focus with strong and continuous contrast transfer over a wide frequency range.

To assess the performance of the Volta phase plate in subtomogram analysis, we obtained subtomogram averages of isolated mammalian ribosomes under constant imaging conditions, either with the Volta phase plate or using conventional defocus-induced phase contrast [6]. Our comparison demonstrates that a given resolution can be obtained with fewer particles using the Volta phase plate, as judged by Fourier shell cross resolution with an external reference structure. This is a significant advantage in cellular tomography, where often only a small number of particles are available. While the subtomogram average obtained close-to focus with the Volta phase plate shows continuous signal transfer over the whole frequency range, subtomogram averages obtained using conventional defocus-induced phase contrast furthermore suffer from lower signal transfer for certain frequency ranges due to oscillations of the contrast transfer function. The alignment accuracy of subtomograms depicting mammalian ribosomes was comparable for both approaches in this study, but we anticipate a much higher benefit of the additional low-frequency contrast provided by the Volta phase plate for smaller complexes with lower contrast. Lastly, our analysis demonstrates that very accurate focusing is the major factor for obtaining subtomogram averages at subnanometer resolution using the Volta phase plate in conjunction with close-to focus acquisition approaches [10].

References:

[1] V Lucic, A Rigort, W Baumeister, J Cell Biol 202 (2013), p. 407-419

[2] M Beck, W Baumeister, Trends Cell Biol. 26 (2016), p. 825-837

[3] JA Briggs, Curr. Opin. Struct. Biol. 23 (2013), p. 261-267

[4] F Forster, R Hegerl, Methods Cell Biol. 79 (2007), p. 741-767

[5] TA Bharat, et al., Structure 23 (2015), p. 1743-1753

[6] M Khoshouei, et al., J. Struct. Biol. 197 (2017), p. 94-101

[7] S Pfeffer, et al., Nat Commun 6 (2015), 8403

[8] F Forster, BG Han, M Beck, Methods Enzymol. 483 (2010), p. 215-243

[9] R Danev, et al., Proc. Natl. Acad. Sci. USA 111 (2014), p. 15635-15640

[10] The authors acknowledge funding from the German Research Council. 